\title{
An automated baseline correction protocol for infrared spectra of atmospheric aerosols collected on polytetrafluoroethylene (Teflon) filters
}

Adele Kuzmiakova et al.

Correspondence to: Satoshi Takahama (satoshi.takahama@epfl.ch)

The copyright of individual parts of the supplement might differ from the CC-BY 3.0 licence. 
S1:

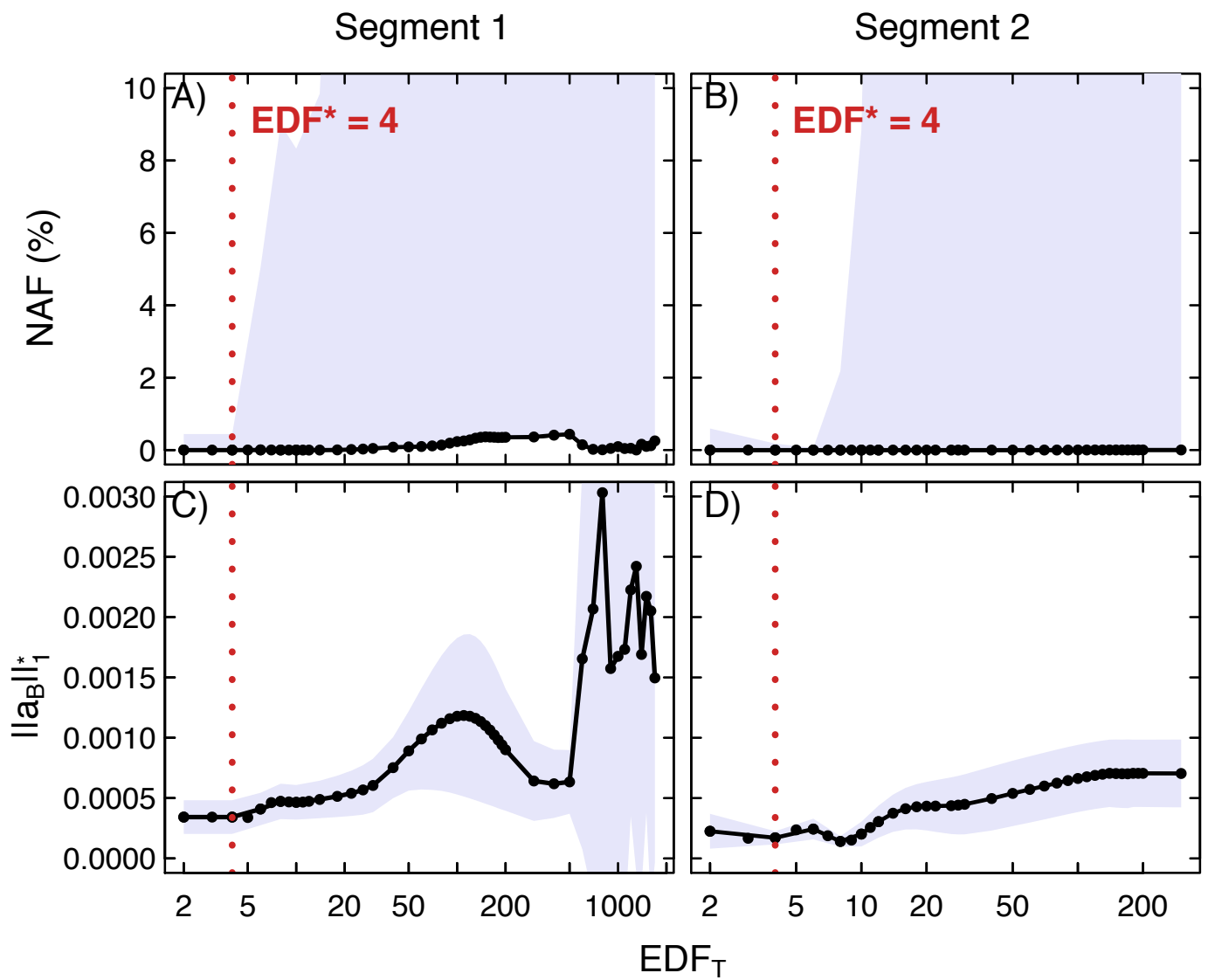

Figure S1: Mean normalized total absolute blank absorbance for $2<=E D F \_T<=n$ where $n=$ 1695 in segment 1 (A) and $n=389$ in segment 2 (B), calculated from 54 IMPROVE 2011 laboratory blank samples (black points). Shaded areas denote two standard deviations from the mean. Median NAF in segment 1 (C) and segment 2 (D) calculated from 794 ambient samples (black points). Lower and upper bounds of shaded areas denote 5th and 65th percentiles. All plots use logarithmic-scale $x$-axis to highlight the behavior in the EDF range we are studying (2 $<=E D F \_T<=12$ ). Black line is drawn to capture the overall trend. 
S2:

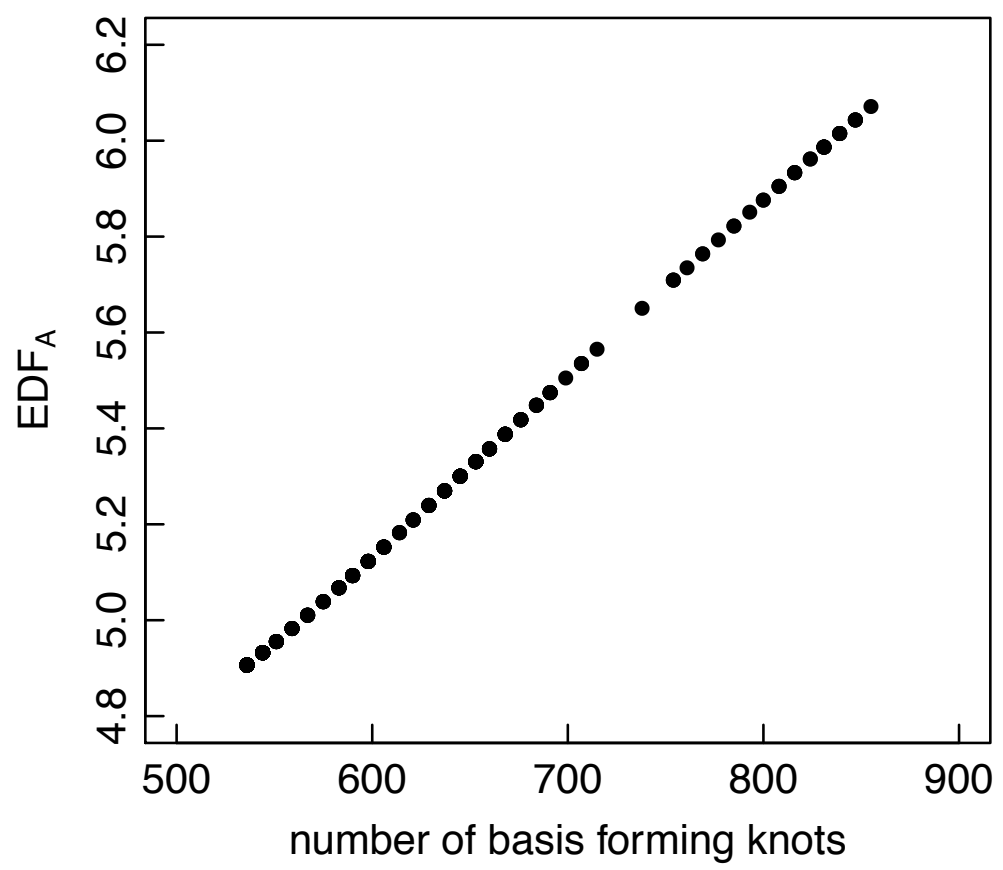

Figure S2: Number of basis forming knots $\left(w \_j=1\right)$ vs EDF_A in segment 1 calculated from 794 ambient samples when EDF_T $=4$. 
S3:

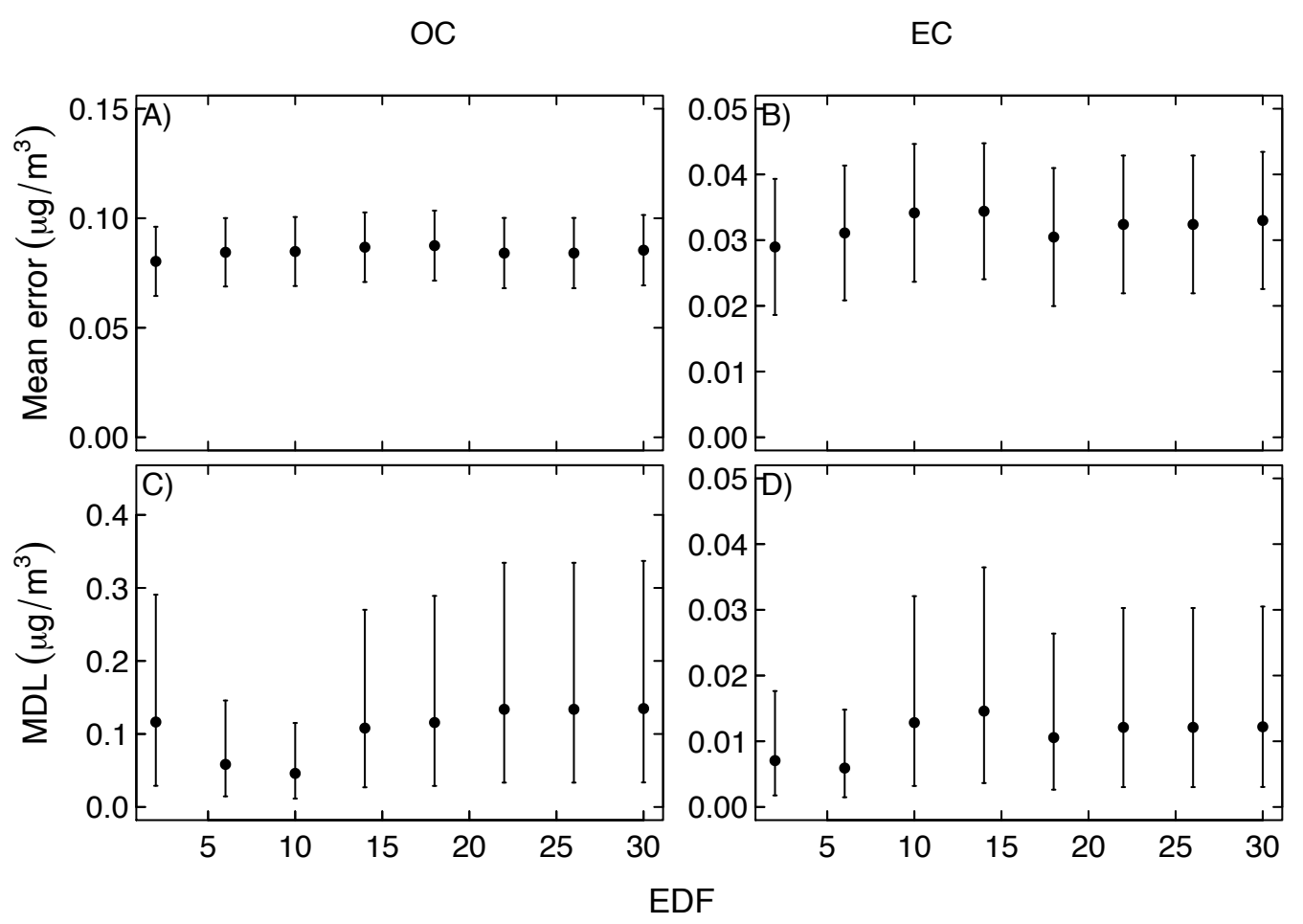

Figure S3: Mean error and MDL metrics for OC predictions ( $A$ and $C$ ) and EC predictions ( $B$ and D) from Test 2011 consisting of spectra corrected by the indicated EDF parameter. The error bars denote the $95 \%$ confidence interval on the estimates. 